\title{
Zu Albert Einsteins 70-tem Geburtstag
}

\author{
M. vON LAUE \\ Kaiser Wilhelm Institut für Physik, Göttingen, Germany
}

$\mathrm{E}_{\mathrm{ft}}^{\mathrm{IN}}$ IN 70-ter Geburtstag ist ein Tag des Rückblicks, für den Jubilar sowohl wie für seine Zeitgenossen. Insbesondere die Gleichaltrigen unter ihnen suchen in ihrer Erinnerung nach den Eindrücken, welche der Jubilar vor 4 bis 5 Jahrzehnten mit seinen ersten Arbeiten auf sie machte, nach den Empfindungen, mit denen sie seine spätere Entwicklung verfolgten. Zustimmung, Zweifel, Opposition, wenngleich vielleicht längst überwunden, werden wieder wach. Und reifer, abgeklärter als ehemals kann das Urtail jetzt sein. Auch persönliche Erlebnisse mit dem Jubilar, Begegnungen mit ihm in guten und bösen Tagen werden seinen Freunden wieder in's Gedächtnis kommen. Aber darüber zu sprechen, ist hier nicht der Platz. Nur auf seine Leistungen werfen wir einen Rückblick.

Einsteins erste Veröffentlichungen betrafen thermodynamische Schwankungen, wie z.B. die Brownsche Molekular-Bewegung und die damit verknüpften Erscheinungen. Für sie interessierten sich zunächst wohl nur die auf demselben Gebiete Arbeitenden; erst allmählich setzte sich diese originelle und elegante Betrachtungsart in der Literatur fest. Aber dann kamen in dem denkwürdigen Jahre 1905 zwei Leistungen, welche im wahrsten Sinne des Wortes Epoche machten.

Der Photo-Effekt, namentlich der befremdende Lenardsche Befund, dass die Höchstgeschwindigkeit der emittierten Elektronen nur von der Frequenz, nicht von der Intensität der Strahlung abhängt, hatte manche Erklärungsansätze gezeitigt, die mit Recht vergessen sind. Einstein gab dafür, Plancks Quantenideen aufnehmend und den Begriff des Lichtquants schaffend, jene so einfache Deutung, die zunächst nur ungläubiges Staunen erregte, sich aber je länger, je mehr als ein Eckstein im Gebäude der Quantentheorie erwies. Eine lange Reihe weiterer quantentheoretischer Arbeiten schloss sich an: die Theorie der spezifischen Wärme, das photochemische Äquivalent-Gesetz, jene Ableitung der Planckschen Strahlungsformel, welche den Bergriff der erzwungenen Emission vielleicht nicht neu schuf, aber erst populär machte, die Bose-Einstein-Statistik; und schliesslich gehört auch der Einstein-de Haas Effekt in diese Reihe. Das Alles sind Leistungen, die allein schon genügten, Einstein zu einem der grössten Physiker aller Zeiten zu stempeln.

Und doch denkt Jeder, der seinen Namen hört, zuerst an die andere grosse Leistung jenes Schicksalsjahres 1905. Im September erschien die Arbeit "Zur Elektrodynamik bewegter Körper." Ein Jahrhundert hindurch hatte die Frage nach dem Bezugssystem für die optischen Erscheinungen die Geister beschäftigt, die Maxwellsche Theorie hatte sie zu einer Frage für die Elektrodynamik erweitert. Seit dem Michelson-Versuch und der berühm- ten Lorentzschen Schrift von 1895 stand sie, mindestens für die Theoretiker, im Vordergrunde des Interesses. H. Poincaré hatte sich um sie bemüht, und erst ein Jahr zuvor hatte H. A. Lorentz eine weitere berühmte Arbeit veröffentlicht, die Alles enthielt, was sich rein mathematisch über die Relativitätsfrage in der Elektrodynamik sagen liess. Einsteins Arbeit ging in dieser Beziehung gar nicht darüber hinaus. Und doch gab sie etwas völlig Neues und Grosses: Die Lorentz-Transformation gab bei ihrem Urheber neben der wahren, absoluten Zeit und dem wahren, absoluten Raum andere Zeiten und Koordinaten, die für die Maxwellschen Gleichungen den "wahren" gleichwertig sind. Aber das schien eine mathematische Merkwürdigkeit. Erst Einstein tat den Schritt, alle diese Zeiten und Koordinatensysteme dem ursprünglichen für gleichwertig $z u$ erklären, für alle Naturvorgänge gleichberechtigt. Dazu gehörte eine Einsicht in das Wesen von Raum- und Zeit-Messung, wie sie vor ihm Niemand besessen hatte.

Das zündete, wie ein Blitz. Weniges macht auf Menschen aller Gattungen solchen Eindruck, als wenn man ihnen an die Vorstellungen von Raum und Zeit rührt. So war das Aufsehen sogleich sehr gross, bedeutete aber nur bei Wenigen, z.B. bei Planck, uneingeschränkte Zustimmung. Durch die Jahrzehnte zog sich, wie hinlänglich bekannt, leidenschaftliche Opposition hin, vermischt mit den denkbar unsachlichsten, sogar politischen Anfeindungen. Aber war es im Fall des "Götterverächters" Aristarch von Samos oder beim Kampfe gegen Kopernikus eigentlich anders?

Aus dieser prinzipiellen Entschiedenheit ergab sich, da sie auch die Mechanik und die thermischen Erscheinungen dem neuen Relativitätsprinzip unterwarf, noch im gleichen Jahre ein Frucht, die zunächst recht unscheinbar aussah, heute aber eine schier erschreckende Popularität erlangt hat: Die Äquivalenz von Energie und Trägheit.

Viele bauten in den folgenden Jahren diese Theorie weiter aus. Max Planck wandte sie auf die Thermodynamik an, Hermann Minkowski gab ihr die elegante, heute unentbehrliche mathematische Einkleidung, indem er das vierdimensionale Kontinuum "Welt" einführte. Ein Erscheinungsgebiet blieb zunächst ausserhalb, die Gravitation. Erst verhältnismässig spät setzten Versuche ein, das Newtonsche Anziehungsgesetż, dieses Musterbeispiel einer unvermittelten Fernwirkung, der Ausbreitung mit endlicher Geschwindigkeit anzupassen, welche die Relativitätstheorie für alle physikalischen Wirkungen fordert. Nur einer dieser Ansätze hat heute noch ein Interesse, die Nordströmschen Theorie von 1912, und zwar als Folie zur "allgemeinen" Relativitätstheorie. 
Die Nordströmsche Gravitationstheorie hatte ihre Qualitäten. Sie postulierte die völlige Unabhängigkeit von Gravitation und elektromagnetischoptischen Vorgängen, sie ergab Newtons Gesetz als eine Näherung für ruhende Körper und ergab, ebenfalls als eine völlig ausreichende Näherung, jene wundersame Gleichheit von träger und schwerer Masse, die empirisch längst anerkannt, aber in der Mechanik ein Anhängsel, kein organisch eingebauter Bestandteil war. Mit alledem war sie in bester Übereinstimmung mit der damaligen Erfahrung. In einem Punkte freilich wich sie von der Beobachtung ab, nämlich bei der Perihelbewegung des Merkur. Aber welcher Physiker hätte wegen dieser einen, durch wenig durchsichtige Störungsrechnung gefundenen, in der Astronomie selbst noch umstrittenen Zahl eine sonst befriedigende, umfassende Theorie verworfen?

Für Einstein aber war das Gesetz der Gleichheit beider Massen mehr wie ein Näherung; das zeigte schon eine Veröffentlichung von 1907. Es war für ihn etwas Fundamentales, etwas so Fundamentales, dass er ihm eine andere, seit jeher allgemein acceptierte und als selbstverständlich angesehene Idee opferte: Die euklidische Massbestimmung im Raume der Physik, und, was damit zusammenhängt, in der Minkowskischen "Welt." Wieder war es ein genialer Einblick in das Wesen von Raum- und Zeit-Bestimmungen, was ihm diese ungeheuerlich scheinende Neuerung ermöglichte. Sie zeitigte aber bald unerwartete, tatsächlich zutreffende Ergebnisse. Nicht nur ergab die "allgemeine" Relativitätstheorie in ihrer allmählichen Entwicklung zwangsläufig den astronomischen Wert für die Perihelbewegnung des Merkur, vielmehr fand die Sonnenfinsternis-Expedition unter Eddington 1919 in der Tat jene Ablenkung des Lichts durch die Sonne, welche diese Theorie als einen Einfluss der Gravitation auf die Fortpflanzung des Lichts forderte. Das war ein Triumph, wie er in der Wissenschaft nur ganz selten vorkommt. Er festigte Einsteins Ruhm für alle Zeiten. Und schliesslich, nach langen Mühen, fanden die Astronomen auch die dritte der von Einstein als beobachtbar erklärten Folgerungen, die Rotverschiebung der Spektrallinien an einer besonderen Klasse von Fixsternen.

Trotzdem ist die allgemeine Relativitätstheorie Einsteins Sorgenkind geblieben; er arbeitet heute noch an ihr. Wir wissen nicht, wie sie sich weiter entwickeln wird; spätere Finsternis-Beobachtungen haben anscheinend die Lichtablenkung nicht ganz in Einklang mit der Berechnung ergeben. Aber die grundlegende Idee, die Einführung nicht-euklidischer Geometrie zur Wahrung des Gesetzes von der Gleichheit beider Massen, dürfte zum gesicherten Bestande der Physik gehören.

Was verehren wir nun bei Einstein am Höchsten? Ich möchte meinen, nicht die gewiss nicht zu unterschätzende mathematische Befähigung, mit der er insbesondere die allgemeine Relativitätstheorie meisterte, nicht die Vielseitigkeit seiner Begabung, nicht einmal die Unbefangenheit gegenüber allen bestehenden Theorieen. Vielmehr steht über dem die schlechthin geniale, unmittelbare, und, wenn sie einmal ausgesprochen ist, so einfache Einsicht in das, was in der Natur wesentlich ist. Sie hat sich in den beiden erwähnten $Z$ weigen gleichmässig bewährt. Aber, um sie wirksam zu machen, musste noch eine Charaktereigenschaft hinzukommen, nämlich eine absolute Ehrlichkeit und ungewöhnlicher Mut der Überzeugung, um gegen uralte, eingefleischte, mächtige Vorstellungen anzukämpfen - zunächst ganz allein. Und diesen Mut, den er auch in dem wohl noch unentschiedenen Kampfe um die Auffassung der Quantenmechanik zeigt, hat er nicht weniger in seinem Verhalten zu den Mächten der Umwelt bewährt. Das werden auch die ihm zugestehen, die ihm in dieser Hinsicht nicht durchweg zu folgen vermögen. Natürlich hat er auch den dafür üblichen Preis bezahlen müssen mit einem unruhigen, manchmal stürmisch bewegten Leben.

Seit einer Reihe von Jahren konnte er nun sein Lebens-Schiff in einen ruhigen Hafen lenken, und die Physiker der ganzen Erde richten am 14 März ihre Gedanken nach Princeton. In dankbarer Verehrung wünschen sie dem Jubilar noch viele Jahre der alten geistigen Klarheit und der alten Freude an der Wissenschaft.

\title{
Einstein's Philosophy of Science
}

\author{
Phillipp Frank \\ Department of Physics, Harvard University, Cambridge, Massachusetts
}

(Received April 29, 1949)

BOUT ten years ago I spoke with Einstein about
the astonishing fact that so many ministers of
various denominations are strongly interested in the
theory of relativity. Einstein said that according to
his estimation there are more clergymen interested in relativity than physicists. A little puzzled I asked him how he would explain this strange fact. He answered, a little smiling, "Because clergymen are interested in the general laws of nature and physicists, very often, are not." 